\title{
Design And Implementation of Remote Video-Audio Communication Module in The Commanding-Dispatching System of Transmitting Stations
}

\author{
Shanshan $\mathrm{Li}^{1, \mathrm{a}}$, Jian Zhou ${ }^{2, \mathrm{~b}}$ and Luying $\mathrm{Jia}^{3, \mathrm{c}}$ \\ ${ }^{1}$ Communication University of China, No.1 Dingfuzhuang East Street, Chaoyang District, Beijing, \\ 100024, P.R. China \\ ${ }^{2}$ Communication University of China, No.1 Dingfuzhuang East Street, Chaoyang District, Beijing, \\ 100024, P.R. China \\ ${ }^{3}$ Communication University of China, No.1 Dingfuzhuang East Street, Chaoyang District, Beijing, \\ 100024, P.R. China \\ alishanshan@cuc.edu.cn , bzhoujian@cuc.edu.cn , 'c85338101@qq.com
}

Keywords: Transmitter, Real Time Media Flow Protocol , Remote video-audio communication , commanding and dispatching

Abstract. In order to improve the overall efficiency of transmitting stations under the new conditions, we need to have a more advanced commanding and dispatching system which is dominated with the practical situation of the work. This article designed the remote real-time video-audio communication module in the commanding and dispatching system. The module has developed on the basis of the RTMFP (Real Time Media Flow Protocol). RTMFP is a P2P communication protocol which was developed by Adobe Company. This module is using the $\mathrm{C} / \mathrm{S}$ architecture. In the module, every transmission station which makes the connection by the way of using P2P can have direct video-audio communication, compared with the traditional way that video-audio data must have server for transit, it effectively reduce the pressure of the server and save the bandwidth resources. It is convenient for the transmission center to arrange working content to every transmission station, the technical experts in the transmission center could have remote technical guidance to every transmission station, and it is easy for the transmission center to carry out the video-audio meeting to all the other transmission station.

\section{Introduction}

With the development of economy and the progress of science and technology, the coverage of radio and television are expanding day by day. The equipment of Radio and television signal transmission are also becoming more and more advanced. The transmitting station integrated monitor and management system which has been built and used has achieved the transfer center to control the operation parameters and working conditions of transmitting stations. In order to improve the overall efficiency of transmitting stations under the new conditions, we need to have a more advanced commanding and dispatching system which is combinated with the practical situation of the work.

\section{The commanding and dispatching system}

The commanding and dispatching system designed and implemented many functions for the daily work of the transfer center such as distributing the tasks to the transmitting stations, receiving the work report from each station, the synchronization and calibration of broadcast schedule for each transmitting station, as well as the real-time audio and video communications between transfer center and transmitting stations. 


\section{key technologies in the System}

\section{Video-Audio Coding technology}

\section{On2VP6}

On2VP6 is the proprietary video format that On2Technologies developed. Adobe and Sun has authorized On2 format to use for Flash and JavaFX, through integration with AdobeFlashPlayer and SunJavaFX, the vedio coding using On2VP6 format can Barrier-free play.On2VP6 can provide excellent compression performance which is better than the SorensonSpark and H.264BaselineProfile provide, and On2VP6 can not only produce higher quality video than AdobeFlashPlayer and SunJavaFX at the same data rate, but also be able to support the use of Alpha channel to create composite video. Under the condition of that the video quality is no significant decline , VP6 is more efficient than h.264 and less to the requirement of operation and transport. The video-audio communication module designed in this paper will adopt the VP6 encoding and transmission.

\section{Speex}

Jean-Marc Valin put forward Speex In 2002, Speex is an open source coding algorithm based on code excitation linear prediction (CELP) coding, it also introduces the function of echo elimination and Anti-shake In the latest version of Speex coding. Speex coding Contains three different sampling rate: $8 \mathrm{kHz}, 16 \mathrm{kHz}$ and $32 \mathrm{kHz}$, three types of sampling frequency respectively represents the narrowband transmission and broadband transmission and ultra-wideband transmission[2]. As a kind of lossy audio codec library, The compression way with Speex can lead to the distortion of audio input signal. Speex can adjust the complexity of the encoder through the selected parameters, under normal circumstances , noise of the complexity of 1 will be $1 \mathrm{db}$ to $2 \mathrm{db}$ higher than the noise of the complexity of 10 , but the higher the complexity, the more requirement of the CPU it needs, users can choose appropriate complexity according to the specific usage scenarios and equipment's processing power. When using Speex codec encoding voice quality increased from 0 to 10 with the increase of the number, at the same time, it will take up more bandwidth for audio signal transmission. Under normal circumstances Speex coding quality defaults to 6. Speex features make it has unique advantages and outstanding performance in the web application. it Is very suitable for development such as voice chat and video meeting applications. video-audio communication module designed in this paper will Using Speex coding to encode the audio signal.

\section{network transmission of the video-audio}

\section{TCP/UDP}

This paper designed and implemented the audio video module based on the RTMFP protocol which is researched and development by the Adobe, RTMFP protocol and RTMP protocol are both development for real-time streaming media transport by Adobe. These protocols with TCP and UDP protocol to cooperate with each other so as to realize the network transport of streaming media data.

\section{RTMP/RTMFP}

RTMP (Real Time Messaging Protocol) which is designed and development by Adobe, it is streaming media proprietary protocols and it is designed for multimedia data transmission through the network between the server and Flash Player. The protocol based on TCP, encapsulated Multiple formats of audio and file type into packets, and each packet is identified by timestamp and payload types, so as to provide reliable data transmission to network multimedia applications.

RTMFP(Real Time Media Flow Protocol) which is designed and development by Adobe, it is communication protocols designed for the users who install the Flash Player to realize directly point-to-point communication. RTMFP uses the P2P technology, makes all nodes which is connected to the network form completely equivalent relation between them, video-audio data in the network will be directly transmitted through point-to-point way without a server for transit, The benefits of this approach is able to effectively reduce bandwidth resources consumption instead of multiple data 
transfer, it is very suitable for large scale network video broadcast live or on demand, and multimedia data transmission business. In order to fully reflect the advantage that RTMFP relative to the RTMP, This article use NS2 to compare them with packet loss rate under different transmission data rate . As shown in figure.1, Loss1. Dat and loss2. Dat represent the packet loss rate of RTMP and RTMFP under different transmission rate. As can be seen from the results, In the process of data rate change, When the rate of close to $250 \mathrm{kbit} / \mathrm{s}$, Network performance change is more intense. RTMFP uses the $\mathrm{P} 2 \mathrm{P}$ technology, makes all nodes which is connected to the network form completely equivalent relation between them, in the RTMP streaming media communications applications, two Flash Player establish a data transmission must pass through the server transfer. RTMFP compared with RTMP, lower packet loss rate, high data transmission efficiency.

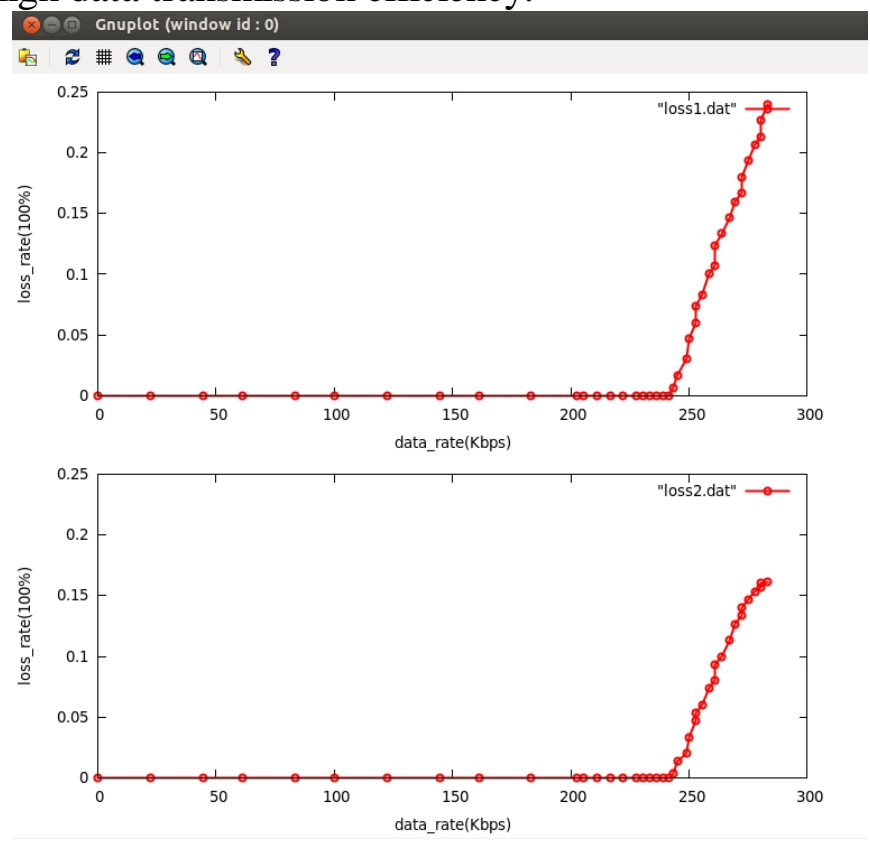

Fig. 1. packet loss rate of RTMP and RTMFP

\section{The realization of the system}

\section{system structure}

Star network structure is a kind of network structure widely used in the enterprise internal network, It is easy to form a network and has easy maintenance, it is advantageous for the server to centralized control on each node in the network, And the star network structure has good scalability which can be convenient to add and remove work nodes, And it also can be flexible adjusted according to the need for application. the star network structure is suitable for Transmission center under the current network conditions, and the advantages of star network structure in video communication are obvious, so the video-audio communication module will use the star network structure which has the center with RTMFP server, As shown in figure.2. 


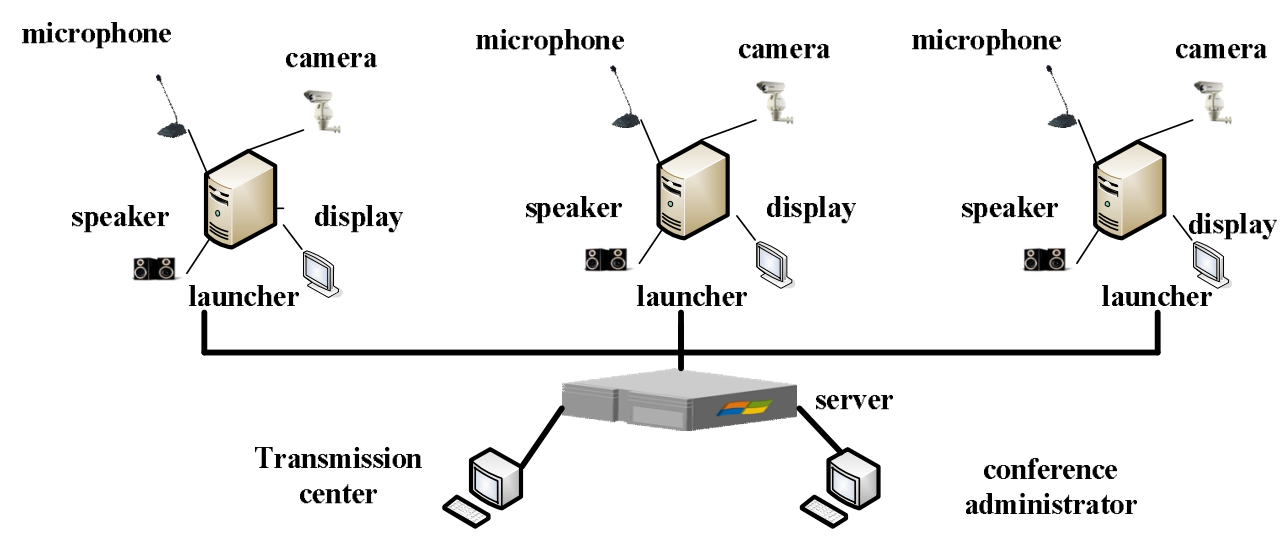

Fig. 2 system structure

\section{Software Realization Method of the system}

\section{The network connection}

The video-audio network transmission is the key of the design of remote video-audio communication module, In this programming it realize video-audio communications in the clients by using the libraries that AS3.0 provide various supporting network transmission on video-audio streaming. This system implement the network connection between the client's and the server's by using flash.net.NetConnection class and create a two-way connection between the client and the server by using NetConnection class. The client can be a Flash Player or AIR applications. The server can be a Web Server, Flash Media Server, or Server that running Flash Remoting application or Adobe state services. This system establish a connection by calling NetConnection().it implement the client and the server's network connection module by using the flash.net.NetConnection class.

\section{transmission of data flow}

This module uses NetStream class to implement the network transmission within the audio, video and data messages. NetStream class opens a one-way flow between client and server by using NetConnection. Adobe AIR and Flash Player 9.0.115.0 select VP6 encoding for video that cameras had collected under the condition of the default.

\section{transmission of Video}

flash.media.Video class Can display real-time video or record video. Video object can sent the real-time video that were captured by the client to the server, then the sever sent this video broadcasting to the other client, so as to realize the transmission in the clients. Video object is an object that can be displayed in the application, the user can determine its operation space by modifying the size of the location of the application that the Video object in .

\section{transmission of Audio}

Remote video-audio communication module obtain audio from other client and play the audio through the speaker by calling constructor named The SoundTransform in the flash media.SoundTransform class . SoundTransform object has two parameters: the value of vol from 0 to 1 , said the volume range from silent to maximum volume, the value of the panning from 1 to 1 , said voice translation from left to right, such as panning set to 1 , the program will only be played from the left speaker, zero value indicates that sound center without translation, by setting the corresponding numerical implementation to them voice broadcast.

\section{System test and result analysis}

We choose three host has a video acquisition device as a test machine in the laboratory environment, use the Wire shark software for a specific host specific agreement to statistical and graphical display of 
real-time data traffic. video-audio communication module use VP6 format in video coding, in the video frame rate to 15 frames per second of the three groups under different video resolution network data traffic generated by the contrast test, so as to calculate and analyz the relationship between the network bandwidth and The video resolution in practical application of transfer center. The two modes are: mode $\mathrm{A}$ is that to establish a two-way video connection between the host and the another host; mode $\mathrm{B}$ is that to establish a two-way video connection between the host and the other two host.

video-audio communication widely used low speed mobile video mode in the launch pad, in the official document of VP6 coding way, it is respectively given high-speed mobile video and low-speed mobile video coding standard Settings under different resolution and frame rate, the low speed mobile video coding standard Settings as been shown in the table 1:

Table 1 Low speed mobile video coding standard setting under VP6

\begin{tabular}{|c|c|c|}
\hline Video resolution (pixel) & Frame rate (fps) & video data rate ( bit/s ) \\
\hline $160 * 120$ & 8 & $40 \mathrm{~K}$ \\
\hline $240 * 180$ & 15 & $100 \mathrm{~K}$ \\
\hline $320 * 240$ & 30 & $180 \mathrm{~K}$ \\
\hline $480 * 360$ & 30 & $350 \mathrm{~K}$ \\
\hline $640 * 480$ & 30 & $600 \mathrm{~K}$ \\
\hline
\end{tabular}

Bandwidth $=$ horizontal resolution $*$ vertical resolution $*$ frame rate $*$ compression ratio in the table 1 , it can be calculated that the compression ratio with VP6 coding in low speed mobile video is about 18 when the frame rate is 30。 In this paper, the design of audio module will unified set frame rate to $15 \mathrm{fps}$, At 15 frames per second by the compression ratio Each resolution video transmission bandwidth can be calculated by the compression ratio under the condition of 15 frames per second. This paper will test the bandwidth respectively in three different resolution : 1280*960, $640 * 480,240 * 180$, the theoretical calculation results of three different resolution as been shown in the table 2, the actual results of three different resolution as been shown in the table 3:

Table 2 theory bandwidth under the three resolution

\begin{tabular}{|c|c|c|c|c|}
\hline \multirow{2}{*}{$\begin{array}{c}\text { resolution } \\
\text { (pixel) }\end{array}$} & \multirow{2}{*}{ mode $\mathrm{A}$} & One host & Two host & total \\
\cline { 3 - 5 } & $2 \mathrm{M}$ & $2 \mathrm{M}$ & $2 \mathrm{M}$ & $4 \mathrm{M}$ \\
\hline $1280 * 960$ & $0.512 \mathrm{M}$ & $0.512 \mathrm{M}$ & $0.512 \mathrm{M}$ & $1 \mathrm{M}$ \\
\hline $640 * 480$ & $0.2 \mathrm{M}$ & $0.2 \mathrm{M}$ & $0.2 \mathrm{M}$ & $0.4 \mathrm{M}$ \\
\hline $240 * 180$ & \multicolumn{3}{|c|}{} \\
\hline
\end{tabular}

Table 3 actual bandwidth under the three resolution

\begin{tabular}{|c|c|c|c|c|}
\hline \multirow{2}{*}{$\begin{array}{c}\text { resolution } \\
\text { (pixel) }\end{array}$} & mode A & \multicolumn{3}{|c|}{ mode B } \\
\cline { 3 - 5 } & & One host & Two host & total \\
\hline $1280 * 960$ & $2.6 \mathrm{M}$ & $2.7 \mathrm{M}$ & $2.8 \mathrm{M}$ & $5.5 \mathrm{M}$ \\
\hline $640 * 480$ & $0.55 \mathrm{M}$ & $0.59 \mathrm{M}$ & $0.61 \mathrm{M}$ & $1.2 \mathrm{M}$ \\
\hline $240 * 180$ & $0.21 \mathrm{M}$ & $0.22 \mathrm{M}$ & $0.23 \mathrm{M}$ & $0.45 \mathrm{M}$ \\
\hline
\end{tabular}


Table 1 and table 2 contrast can be seen, in the 640*480 resolution and 240*180 resolution , Actual test results was consistent with the theoretical calculation results, in the $1280 * 960$ resolution, error between the actual test results with the theoretical calculation results is a little big, This is because compression ratio was calculated as a constant in the theoretical calculation, but in the practical application of video transmission, the mobile of the video elements can cause the compression ratio dynamic change, which affect the bandwidth of the video transmission.

From the actual test results, it can be concluded that with the increase of resolution, bandwidth of real-time video transmission occupies is increasing, with an increase in the number of video connections, the bandwidth consumption is also increased. At present, the bandwidth of the transmission center can reach $100 \mathrm{M}$, it can be able to meet the requirements of transmitting multiple hd video data . the bandwidth of the launch station is $2 \mathrm{M}$, it can meet the requirements of single hd video transmission to the center. In the practical application of the monitoring center, therefore, it should adjust the video resolution according to the scale and bandwidth of each terminal, so as to gain better video transmission.

\section{Conclusions}

In the module, every transmission station which makes the connection by the way of using P2P can have direct video-audio communication, compared with the traditional way that video-audio data must have server for transit, it effectively reduce the pressure of the server and save the bandwidth resources. It is convenient for the transmission center to arrange working content to every transmission station, the technical experts in the transmission center could have remote technical guidance to every transmission station, and it is easy for the transmission center to carry out the video-audio meeting to all the other transmission station.

Although this module achieve the function of video-audio communication, but there are many ways remains to be further improvement and optimization. In addition ,the security of this module should be strengthen.

\section{References}

[1] Ye, J.H., Yang, C.Y., Zhou, J., (2008). Research of Radio and Television Transmitter Fault Diagnosis Expert System, Radio\&TV Brosdcast Engineering, In Chinese.8,48-51. 2.

[2] S.Khattak,R.Hamzaoui,S.Ahmad,P.Frossard.Fast encoding techniques for Multiview Video Coding[J]. Image Communication, 2013, Vol.28 (6):569-580.

[3] Wu, J.L, research and design based on the IP network video conference system [D]. In Chinese.South China University of Technology,2006.

[4] Li,Z.C,.IP Modeling and research on distortion for video transmission over IP data optical natworks [D]. In Chinese.Beijing University Of Posts And Telecommunications, 2009.

[5] Gao, Q.T, Liu, J.L, Zhang, T.T, RTMFP protocol in the application of P2P video communication [J]. In Chinese.computer systems applications,2012,21(3):176-177.

[6] Peng, Z., Wei,H.W., Bo Z., Min,Z.L., Hiding information in MPEGsequences by using of B-Frames[J]. In Chinese.Wuhan University Journal of Natural 